\title{
Perturbed Pattern of Catecholamine-Containing Neurons in Mutant Drosophila Deficient in the Enzyme Dopa Decarboxylase
}

\author{
Vivian Budnik, ${ }^{*}$ Linda Martin-Morris, $\dagger$ and Kalpana White $\nmid$ \\ *Biophysics Program, Brandeis University, and †Department of Biology, Brandeis University, Waltham, \\ Massachusetts 02254
}

We have initiated a study of catecholamine-containing neurons in Drosophila melanogaster because of the potential, with this organism, to perturb catecholamine metabolism using genetic tools. The major objectives of this study were (1) to define the pattern of catecholamine-containing neurons and (2) to determine the effect of the absence of dopa decarboxylase (DDC) enzyme activity on the catecholamine-containing neurons.

We chose to analyze the catecholamine-containing neurons in the ventral ganglion of the larval CNS. To define the catecholamine-containing neurons, CNSs were dissected and reacted with glyoxylic acid. The catecholamine histofluorescence (CF) neuronal pattern (normal-CF neurons) in the wild-type ventral ganglion is stereotypic. In the mutant ventral ganglia, in the absence of DDC enzyme activity, most normal-CF neurons still exhibit $C F$, probably indicating the presence of accumulated L-dopa. Interestingly, in the mutant CNSs, additional novel neuronal subsets also exhibit CF. Analysis of CNSs from early developmental stages revealed that the novel-CF neurons become fluorogenic earlier than the normal-CF neurons in the mutant CNS.

To determine whether neuronal subsets, in addition to the normal-CF, neurons are able to sequester catecholamines, CNSs from wild-type larvae were incubated in exogenous catecholamine (L-dopa or dopamine). Incubations in L-dopa or dopamine revealed normally nonfluorogenic neurons that are able to take up the amine and become fluorogenic. Among the neurons able to sequester $\mathrm{L}$-dopa or dopamine are subsets that are similar to the novel-CF neurons in the mutant CNS. This similarity is best characterized by a major novel-CF neuronal cluster in the subesophageal-thoracic region. These results suggest that in the absence of DDC activity, subsets of normally nonfluorogenic neurons capable of sequestering $L$-dopa or dopamine accumulate the fluorogenic catecholamine. Hypotheses that might explain the mode of accumulation of the catecholamine within the novel$C F$ neurons are considered.

Within the last decade it has become increasingly possible to study the chemical anatomy of the nervous system, employing histochemical and immunohistochemical methods (e.g., Hökfelt et al., 1984). Anatomical descriptions of the vertebrate and invertebrate systems focus on the localization of neurotransmitters, the enzymes utilized in the synthesis of neurotransmitters, and neuropeptides. A striking but not unexpected fea-

\footnotetext{
Received Mar. 11, 1986; revised June 23, 1986; accepted July 1, 1986.

We appreciate the helpful comments of Eve Marder and Michael Nusbaum during the preparation of this manuscript. We wish to thank Ana Maria Valles for willingly providing invaluable help at many levels during the course of this work and for stimulating discussions. This work was supported by NIH Grant NS 23510 . V.B. is a predoctoral trainec, supportcd by a Brittner fellowship at Brandeis University.

Correspondence should be addressed to Kalpana White at the above address.

Copyright (c) 1986 Society for Neuroscience $0270-6474 / 86 / 123682-10 \$ 02.00 / 0$
}

ture of the emerging picture is the stereotypic distribution within the CNS of a given chemical messenger. The advances in chemical neuroanatomy have contributed to the clarification of the chemical organization of the CNS. But at the same time, the discovery of a large number of molecules with putative neurotransmitter-like or neuromodulator-like functions has brought about a new awareness of the complex overall organization.

Presumably, for proper operation of the CNS, programmed differentiation of neurons and continuous overall regulation of the synthesis of a large array of molecules are essential. During development, different neurons develop the capacity to synthesize and use 1 or more specific chemical signals, resulting in the final pattern. The mechanisms responsible for the exquisite control of cellular differentiation and continued metabolic regulation operating in the nervous system are largely unknown.

It is indeed possible that some of the transmitter or modulator molecules might provide specific signals essential for the proper differentiation or maintenance of normal functions in some subsets of neurons. The recent work of Kater and co-workers on the regenerating neurons in cell cultures of the snail Helisoma implicate the biogenic amine transmitters, dopamine and serotonin, as regulators of neurite outgrowth (Haydon et al., 1984; McCobb et al., 1985; Mercier et al., 1985).

Genetically mutant animals incapable of synthesizing specific transmitters provide in vivo model systems in which to study the effect of the absence of the transmitter on the development and function of the nervous system (Greenspan, 1980; Greenspan et al., 1980; Vallés and White, 1986). The study of neurochemical changes in neurons that are directly affected by the mutation, and of other associated chemical and morphological changes that might occur in the mutant CNSs may potentially provide insights when the primary biochemical lesion is well defined.

We have been studying the biogenic amine systems in the fruit Hy, Drosophila melanogaster, as it is possible to generate animals that are genetically deficient in dopa decarboxylase (DDC) enzyme activity and thus are presumably deficient in serotonin and catecholamines (Livingstone and Tempel, 1983; Vallés and White, 1986). In previous work, we studied the differentiation of serotonin-containing neurons in their transmitterless state in $D d c$ mutant CNSs. Neurons normally committed to the serotonin differentiation pathway proceeded to express selective serotonin-uptake properties in the absence of DDC enzyme activity (Vallés and White, 1986). In this paper, we extend our investigation of CNSs deficient in DDC enzyme activity to catecholamine-containing neurons.

\section{Materials and Methods}

\section{Fly cultures and strains}

All fly strains were raised at $25^{\circ} \mathrm{C}$ on standard medium. The mutant chromosomes used are listed below. For more complete descriptions of 

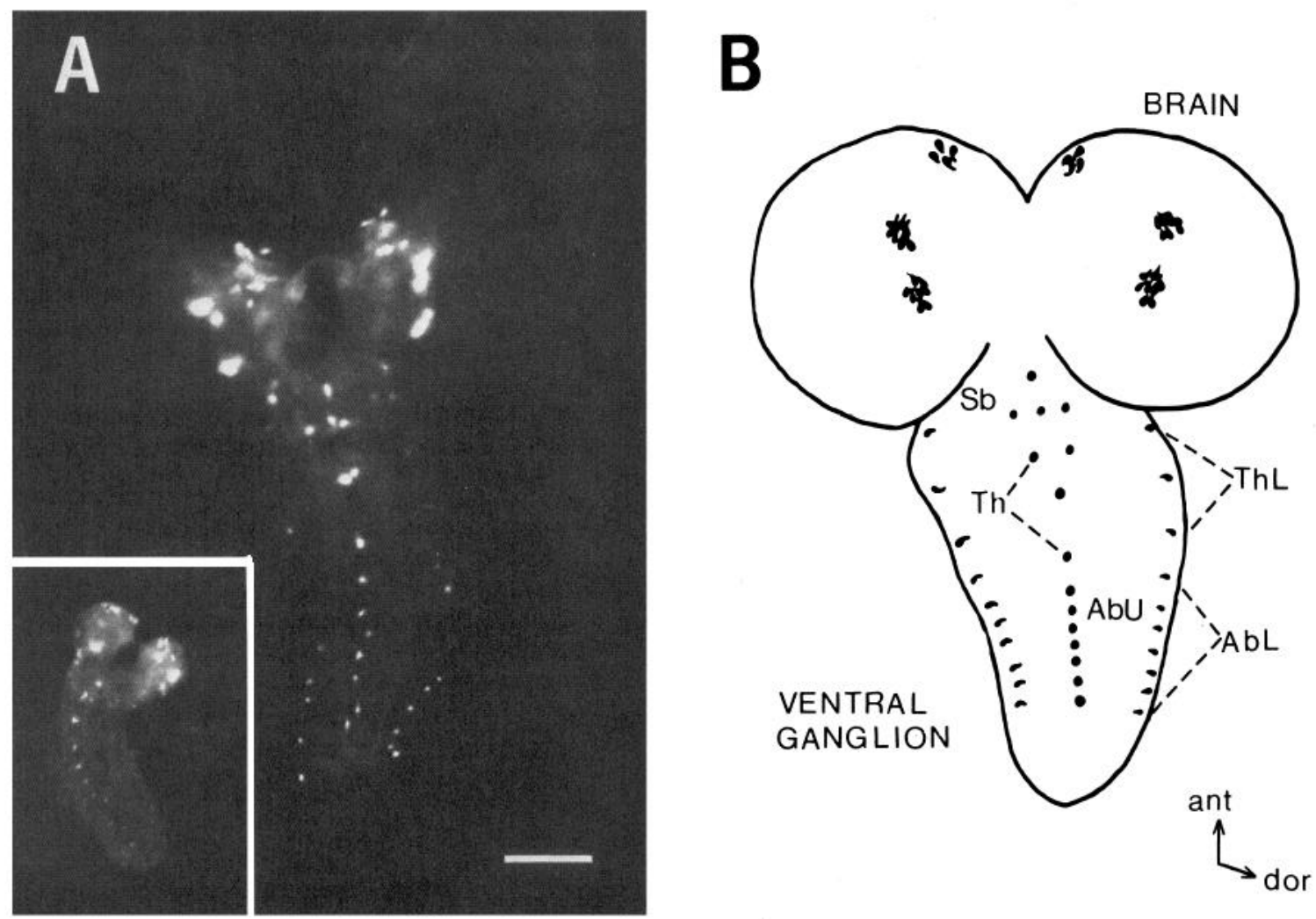

Figure 1. Normal-CF neurons in the larval wild-type ventral ganglion. $A$, Photomicrograph of a whole-mount preparation of a third instar wildtype CNS. For schematic representation, see $B$. In this plane, most of the Sb-Th neurons and all the AbU neurons can be resolved. Also some of the AbL neurons can be readily observed. Insert shows a first instar CNS dissected from a newly hatched larva. In this photographic plane, only some of the normal-CF neurons in the first instar larval CNS are observed. The neurons observed are from the Sb-Th region and some of the anterior AbU neurons. Bar, $30 \mu \mathrm{m}$. B, Schematic tracing of the normal-CF neurons adapted from whole-mount preparations of glyoxylic acidreacted, wild-type larval CNSs. The histofluorescence in the neuropil and primary processes of the neurons are not drawn. ant, Anterior; $A b L$, abdominal lateral neurons; $A b U$, abdominal unpaired neurons; dor, dorsal; $S b$, subesophageal paired and unpaired neurons; $T h$, thoracic paired and unpaired neurons; $T h L$, thoracic lateral neurons.

mutations, see Vallés and White (1986). The wild-type strain used was Canton S.

$D f(2 L) 130$. A cytologically visible deletion, entirely removing the gene Ddc (Wright et al., 1976).

$D d c^{n 27}$. A $2 \mathrm{~kb}$ deletion removing the two $5^{\prime}$ exons of $D d c$ and $0.5 \mathrm{~kb}$

of 5' flanking DNA (Gilbert et al., 1984).

$\mathrm{CyO}$. A second chromosome balancer.

SM6. A second chromosome balancer.

\section{Generation of animals deficient in the gene Ddc}

The genetic scheme and specific crosses used to generate animals that were deficient in DDC activity $(D f D d c)$ are detailed in Vallés and White (1986). The genetic crosses were as follows:

$D f(2 L) 130 / C y O \times D d c^{n 27} / C y O$ or $D d c^{n 27} / S M 6 \times D d c^{n 27} / S M 6$. Twentyfive percent of the progeny of these crosses were $D d c$-deficient (genotype: $D f(2 L) 130 / D d c^{n 27}$ or $\left.D d c^{n 27} / D d c^{n 27}\right)$.

Most $D f D d c$ mutant embryos die at the embryo larval boundary and do not hatch. However, they are fully developed and if liberated from the embryonic membranes about $40 \%$ are able to undergo larval development (Vallés and White, 1986). The mutant pharate larvae can be readily recognized in late embryogenesis $(20-22 \mathrm{hr})$ because of their incompletely sclerotized mouth hooks. Naturally or mechanically hatched mutant animals were reared in a special medium. The details of this procedure are described elsewhere (Vallés and White, 1986).

\section{Glyoxylic acid-induced histofluorescence}

To visualize the catecholamine-containing neurons, the glyoxylic acid histofluorescence method, adapted for invertebrate tissue, was used (Flanagan, 1983). The larval CNSs were dissected in $\mathrm{Mg}^{2+}$-rich, ice- cold Drosophila Ringer's solution ( $\mathrm{NaCl}, 110 \mathrm{~mm} ; \mathrm{KCl}, 4.7 \mathrm{~mm} ; \mathrm{MgCl}_{2}$, $\left.20 \mathrm{~mm} ; \mathrm{CaCl}_{2}, 1.8 \mathrm{~mm} ; \mathrm{KH}_{2} \mathrm{PO}_{4}, 0.74 \mathrm{~mm} ; \mathrm{Na}_{2} \mathrm{HPO}_{4}, 0.35 \mathrm{~mm}\right)$. The dissected CNSs were transferred to a glass microscope slide, gently blotted, and incubated in approximately $0.1 \mathrm{ml}$ of the glyoxylic acid solution [5\% glyoxylic acid (Mallinckrodt), $0.2 \mathrm{M}$ sucrose in $\mathrm{Ca}^{2+}$-free Drosophila Ringer's solution ( $\mathrm{NaCl}, 110 \mathrm{~mm} ; \mathrm{KCl}, 4.7 \mathrm{~mm} ; \mathrm{MgCl}_{2}, 1.80$ $\mathrm{mm} ; \mathrm{KH}_{2} \mathrm{PO}_{4}, 0.74 \mathrm{~mm} ; \mathrm{Na}_{2} \mathrm{HPO}_{4}, 0.35 \mathrm{~mm}$ ), adjusted to $\left.\mathrm{pH} 7.0\right]$. The incubations were carried out in a moist, dark chamber for $1 \mathrm{hr}$ at room temperature. The solution was then blotted away from the tissue and the samples were dried over $\mathrm{Ca}_{2} \mathrm{SO}_{4}$ overnight in the dark. Thoroughly dried tissues were covered with a drop of heavy mineral oil and flashheated for $2 \mathrm{~min}$ at $80^{\circ} \mathrm{C}$ to catalyze the second step of the glyoxylic acid reaction. The samples were viewed in epifluorescence in a Zeiss inverted microscope (Zeiss 487705) equipped with the appropriate excitation and barrier filters. Samples were stored in the dark at $4^{\circ} \mathrm{C}$. Under these conditions, cells remained fluorescent for up to 3 months.

\section{Preloading with catecholamines}

Larval CNSs were dissected in Drosophila Ringer's $(\mathrm{NaCl}, 130 \mathrm{~mm}$; $\mathrm{KCl}, 4.7 \mathrm{~mm} ; \mathrm{CaCl}_{2}, 1.8 \mathrm{~mm} ; \mathrm{KH}_{2} \mathrm{PO}_{4}, 0.74 \mathrm{~mm} ; \mathrm{Na}_{2} \mathrm{HPO}_{4}, 0.35 \mathrm{~mm}$ ). A $10^{-2} \mathrm{M}$ stock solution of each amine tested-L-dopa, dopamine, and norepinephrine-was made up into Ringer's. Tyrosine was dissolved in EtOH at a final concentration of $10^{-1} \mathrm{M}$. Dissected CNSs were incubated individually in concentrations ranging from $10^{-3}$ to $10^{-7} \mathrm{M}$ amine in Ringer's. Incubations were carried out for $30 \mathrm{~min}$ in the dark at room temperature, with 1 change of the amine solution after the first $15 \mathrm{~min}$. Approximately $0.1 \mathrm{ml}$ of the solution was used per CNS. After $30 \mathrm{~min}$ incubation time, the solution was removed and the tissue washed with Ringer's 4 times over a period of $40 \mathrm{~min}$ at room temperature. The final wash solution was replaced with the glyoxylic acid solution and reacted as described above. 

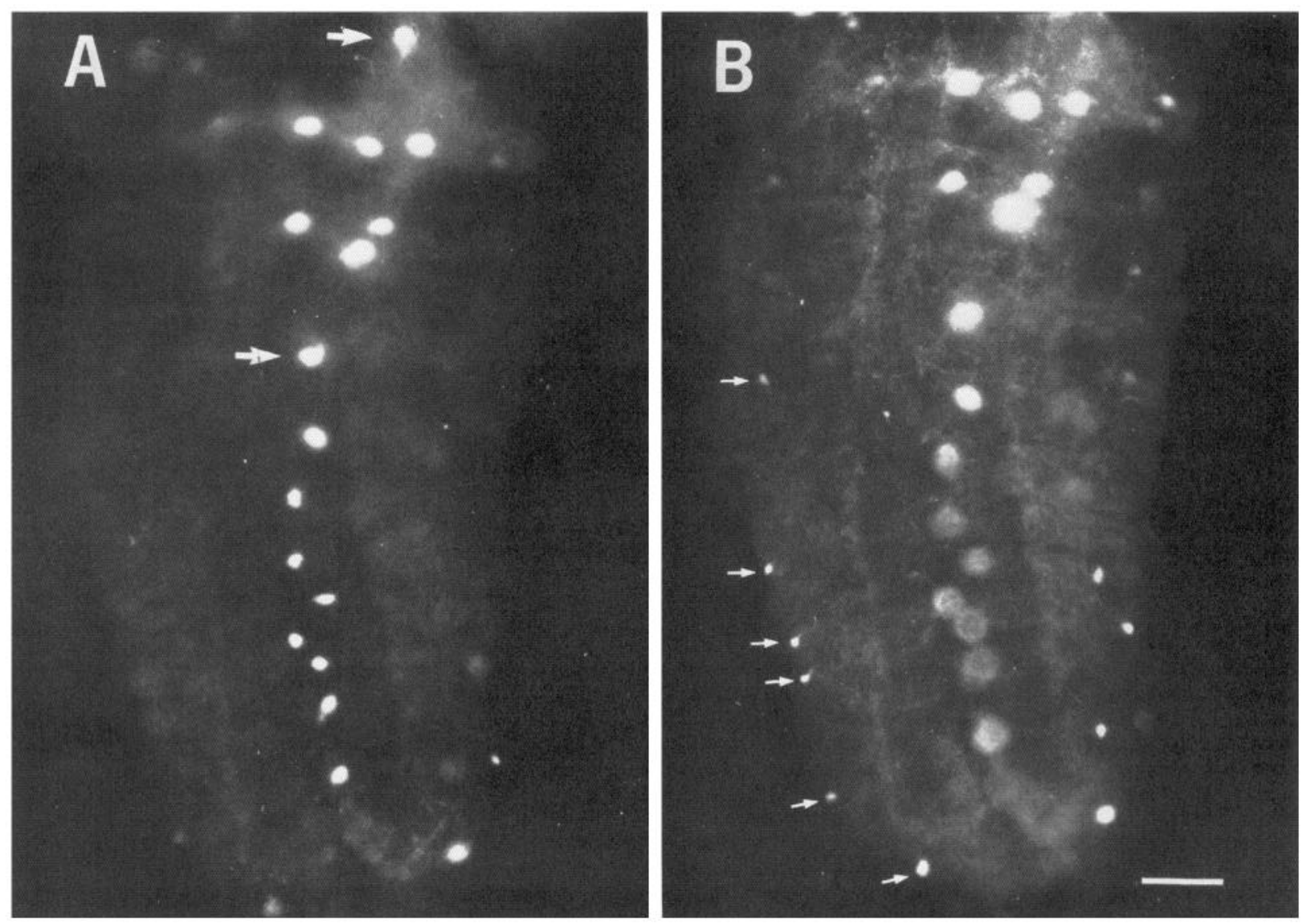

Figure 2. Normal-CF neuronal subsets in the ventral ganglion. A, Photomicrograph of a ventral plane showing the Sb-Th cluster (delineated by the 2 white arrows) and the $\mathrm{AbU}$ neurons located posterior to the second arrow. B. Same whole-mount as in $A$, but the plane of the photomicrograph represents some of the dorsally located lateral neurons. The white arrows point to the left AbL neurons; several of the right neurons are also observed. Bar, $20 \mu \mathrm{m}$. Axes, same as Figure 1.

\section{Results}

Catecholamine-containing neurons in Drosophila larval CNS Glyoxylic acid-induced histofluorescence. Glyoxylic acid converts certain monoamines to highly fluorescent derivatives (Lindvall and Björklund, 1974; Lindvall et al., 1974). The fluorescent condensation products allow the histochemical localization of monoamine-containing neurons (Lindvall and Björklund, 1974). Recently the glyoxylic acid method has been successfully employed to localize monoamine-containing neurons in invertebrate CNSs (Flanagan, 1983; Lent, 1982). There are 3 points regarding the specificity of the reaction that are pertinent to the interpretations of our results:

1. Both catecholamines and serotonin can react with glyoxylic acid to form fluorophores; the fluorescence specific to catecholamines is blue-white and is relatively stable, whereas the fluorescence specific to serotonin is yellowish and unstable (Lent, 1982; Lindvall et al., 1974). The pH optima for the catecholamine and serotonin reactions are distinct (Lindvall et al., 1974). In this study, we used conditions that favored the catecholamine reaction (neutral $\mathrm{pH}$ ), and only the blue-white fluorescence specific to catecholamine-containing neurons was observed. These neurons will be referred to as "CF neurons."

2. Glyoxylic acid reacts with several compounds in the catecholamine pathway. A major disadvantage of the method is that it is virtually impossible to distinguish between L-dopa, dopamine, and norepinephrine since the fluorophores they yield have similar spectral characteristics (Lindvall et al., 1975). Therefore, positive identification of the catecholamine responsible for the fluorescence is not possible using this method alone.

3. In the histochemical application of this method for visualizing catecholamine-containing neurons, it is generally assumed that the amine responsible for the fluorescence is dopamine or norepinephrine. L-Dopa, the precursor to dopamine, does not make a significant contribution to the fluorescence, as the intracellular pools of L-dopa are negligible in comparison to the dopamine or norepinephrine pools (Klemm, 1980). The fluorescence yield from octopamine is very low compared to that of dopamine or epinephrine; therefore, the method is not effective in detecting octopamine-containing neurons.

Larval CF pattern. A stereotypic pattern of CF neurons is observed after the glyoxylic acid treatment of intact CNSs dissected from the Drosophila larvae (Budnik et al., 1985). Figure $1 A$ is a photomicrograph of a glyoxylic acid-treated whole-mount preparation. Figure $1 B$ is a schematic representation of a third instar larval CNS onto which the somata of the CF neurons are drawn. The tracing is adapted from glyoxylic acid-treated CNS whole-mounts similar to the one shown in Figure $1 \mathrm{~A}$.

$\mathrm{CF}$ neurons were observed in the paired brain hemispheres and in the ventral ganglion. In the brain lobes, 3 bilaterally symmetrical clusters of 4-6 CF neurons each were routinely observed. In this paper we focus on the $\mathrm{CF}$ neurons observed in the ventral ganglion, as the regularity of the pattern allows direct comparison between different samples. The ventral ganglion of the Drosophila larva is a compound ganglion composed 


\begin{tabular}{|c|c|c|c|c|c|c|}
\hline \multirow{3}{*}{$\begin{array}{l}\text { Sample } \\
\text { no. }\end{array}$} & \multicolumn{2}{|c|}{ Sb-Th } & \multicolumn{2}{|c|}{$\begin{array}{l}\mathrm{Ab} \\
\text { (Maximum expected) }\end{array}$} & \multirow{3}{*}{$\begin{array}{l}\text { Th } \\
\text { L } \\
(6)\end{array}$} & \multirow{3}{*}{$\begin{array}{l}\text { Total } \\
\text { (36) }\end{array}$} \\
\hline & $\mathrm{U}$ & $\mathrm{P}$ & $\underline{\mathrm{U}}$ & $\mathrm{L}$ & & \\
\hline & (4) & (4) & (8) & (14) & & \\
\hline 1 & 4 & 4 & 7 & 14 & 5 & 34 \\
\hline 2 & 4 & 4 & 7 & 14 & 4 & 33 \\
\hline 3 & 4 & 4 & 7 & 14 & 4 & 33 \\
\hline 4 & 4 & 4 & 7 & 14 & 4 & 33 \\
\hline 5 & 4 & 4 & 8 & 12 & 4 & 32 \\
\hline 6 & 4 & 4 & 8 & 14 & 2 & 32 \\
\hline 7 & 4 & 4 & 7 & 13 & 4 & 32 \\
\hline 8 & 4 & 4 & 7 & 14 & 2 & 31 \\
\hline 9 & 4 & 4 & 6 & 13 & 3 & 30 \\
\hline 10 & 4 & 4 & 7 & 14 & 0 & 29 \\
\hline 11 & 4 & 4 & 7 & 14 & 0 & 29 \\
\hline 12 & 4 & 4 & 7 & 11 & 0 & 28 \\
\hline 13 & 4 & 4 & 5 & 10 & 0 & 27 \\
\hline
\end{tabular}

of subesophageal (Sb), thoracic ( $\mathrm{Th}$ ) and abdominal (Ab) neuromeres. The pattern of CF neurons observed in the wild-type ventral ganglion can be divided into 3 subgroups of neurons for clarity of presentation. These 3 neuronal subgroups will be referred to as the normal-CF neuronal set in this paper. They are as follows:

1. Medial subesophageal-thoracic neurons. This region contains a total of $8 \mathrm{CF}$ somata, located in the ventral cellular rind. There are 4 unpaired medial neurons (U) and 2 pairs of medially located neurons (P). At present, the boundary between the subesophageal and thoracic regions is not clear. Tentatively, the anterior pair of neurons and the 2 anterior unpaired medial neurons are localized to the subesophageal ganglion, and the posterior pair and the posterior unpaired medial neurons are localized to the thoracic neuromeres. This anatomical subdivision is supported by the observation that a medial pair and 2 unpaired neurons are observed in the subesophageal ganglion in the adult brain (V. Budnik, unpublished observations). Figure $2 A$ depicts the 4 paired and 4 unpaired neurons in the medial $\mathrm{Sb}$ - Th region.

2. Abdominal unpaired medial row. A row of single unpaired $\mathrm{CF}$ neurons ( $\mathrm{AbU}$ ) was observed along the ventral midline in the abdominal region. Presumably, each of the anterior abdominal neuromeres contains 1 unpaired CF neuron (Fig. $2 A$ ). In most preparations, 7 neurons were observed, although, on occasion, 8 neurons were observed.

3. Paircd latcral row. A single CF soma was observed in each of the 3 thoracic hemineuromeres (ThL) and in the anterior 7 abdominal hemineuromeres $(\mathrm{AbL})$. The location of the lateral neuronal somata was dorsal to the medial unpaired neurons. The neuronal cell bodies along the lateral row appeared to be smaller than the medial neuronal cell bodies. This pattern was bilaterally symmetrical (Fig. $2 B$ ).

Table 1 represents a count of CF somata in the ventral ganglion as observed in individual wild-type whole-mount CNS samples from the third instar larvae (13 samples). The first row represents the maximum observed number of somata for each of the neuronal sets, which adds up to a total of $36 \mathrm{CF}$ neurons per ventral ganglion. The individual samples are listed in descending order of total neurons seen. The total number of neurons observed in an individual ganglion varied between 27 and 34 , with a mean of 31 . The major point made in Table 1 is that the endogenous normal- $C F$ pattern is robust. The 8 expected

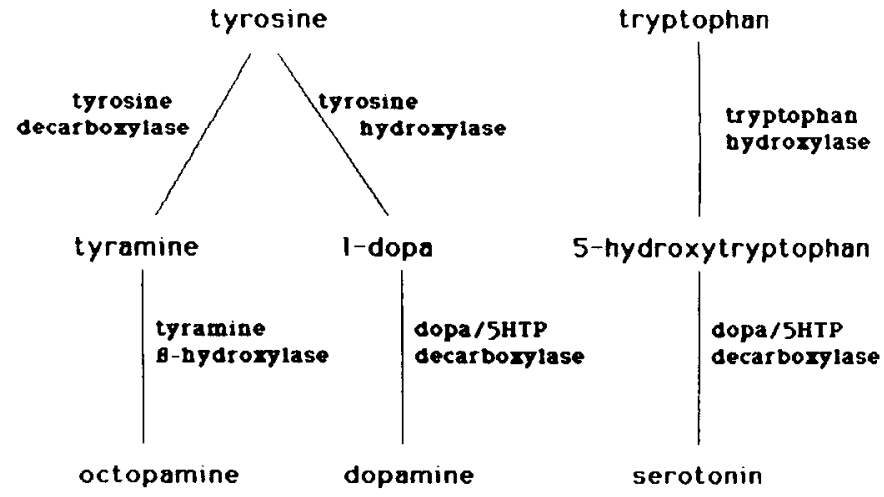

Figure 3. Pathway of monoamine biosynthesis in Drosophila, as suggested by Livingstone and Tempel (1983).

Sb-Th neurons were always observed. The majority of the AbU and $\mathrm{AbL}$ neurons were observed routinely. The ThL neurons were not observed in many samples. The variability between samples was primarily due to the absence of certain neurons. We believe that 2 factors contributing to the absence of certain CF neurons were that (1) the cell bodies are located superficially in the cellular rind; if the sheath was damaged, some neurons may have been lost during the procedure, and (2) the catecholamine concentration within some of these neurons may be close to the threshold of detection under the experimental conditions.

The pattern of CF neurons described above for the CNS of a third instar larva is already present in the newly hatched larvae (see insert, Fig. 1 $A$ ), and, al least in its more global aspects, is maintained throughout the larval stages.

\section{Df Ddc CNSs of third instar larvae exhibit a perturbed pattern of $C F$}

Animals genetically deficient in DDC activity can live up to 6$7 \mathrm{~d}$ and develop as far as the late larval stages (Vallés and White, 1986). Figure 3 depicts the biochemical pathway used in the synthesis of serotonin, dopamine, and octopamine, as suggested by Livingstone and Tempel (1983). The absence of DDC activity is expected to result in the absence of dopamine and serotonin, as the decarboxylation of L-dopa and 5-hydroxytryptophan will be blocked. In the case of the CF neuronal pattern of the mutant CNSs, several scenarios are theoretically possible: (1) CF neurons are not observed; (2) the CF pattern remains the same as the normal-CF pattcrn; (3) the CF pattern in the mutant is different from the normal- $C F$ pattern. It is important to remember that glyoxylic acid reacts with $\mathrm{L}$-dopa (as well as with dopamine and norepinephrine) and that at least 1 possible outcome of the absence of DDC activity may be the accumulation of L-dopa to levels not normally attained by this precursor in the neurons.

In order to investigate the effect of the absence of DDC activity on catecholamine distribution in the CNS, we generated and reared animals deficient for the gene $D d c$, as described in Materials and Methods (these animals will be referred to as mutant or $D f D d c$ ). The CF neuronal pattern observed in the $D f D d c$ CNSs of third instar larvae differed from the wild-type CF neuronal pattern. The most noticeable aspect of CF observed in the mutant ventral ganglion was the appearance of several classes of fluorescent neurons not previously observed in the wild-type ventral ganglion. First, the mutant $\mathrm{CF}$ pattern was examined in order to define any distinctive aspects observed only in the mutant CNSs; these constitute the novel-CF neurons. It was difficult to define all features of the mutant CF pattern because of a larger population of fluorescent neurons and the variability in the intensity of fluorescence of certain neurons. 
Figure 4. CF neuronal pattern in the $\mathrm{Sb}-\mathrm{Th}$ region of the $D f D d c$ and wildtype third instar larval CNSs. The prints are a negative image of a fluorescence micrograph. $A$, Photomicrograph showing the novel-ST cluster in the mutant $D f D d c$ CNS. White arrows point to the limits of the novel$S T$ cluster. Black arrowheads point to 2 of the lateral neurons. $B$, Photomicrograph showing the normal- $C F$ pattern in the wild-type CNS. White arrows delineate the normal-CF neurons in the medial $\mathrm{Sb}$ - $\mathrm{Th}$ region. Compare the $\mathrm{CF}$ neurons in the region between the arrows in $A$ and $B$. Bar, $20 \mu \mathrm{m}$. Axes, same as Figure 1.

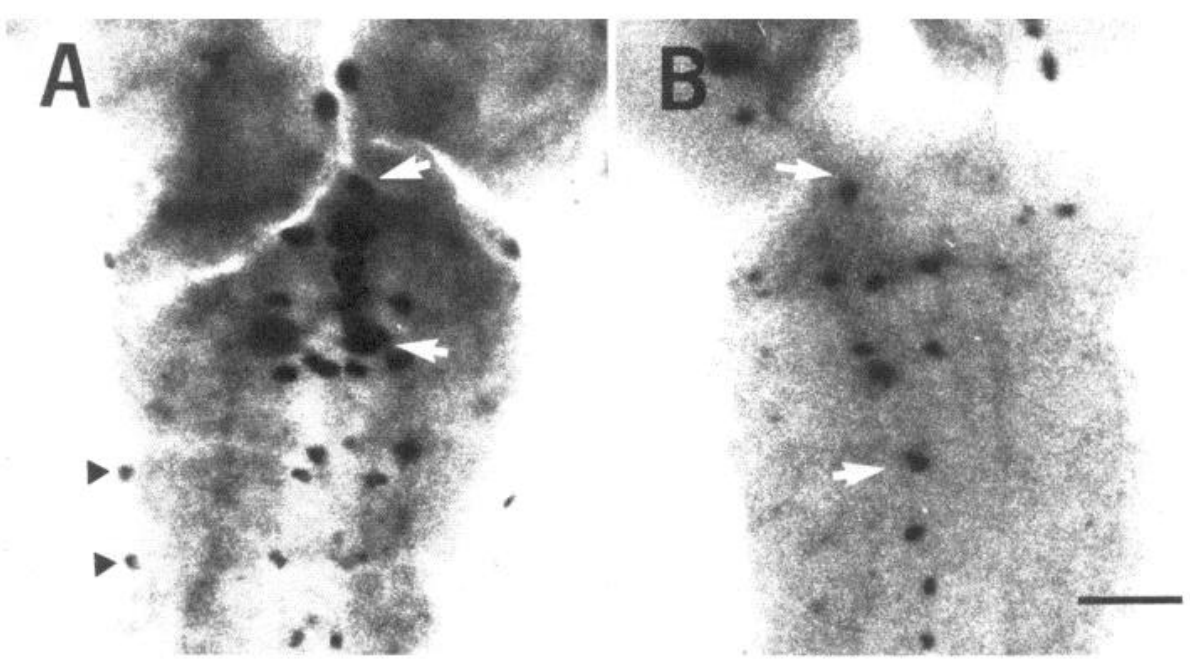

Nevertheless, a novel-CF neuronal set not observed in wild-type was found in the Sb-Th region in all mutant $\mathrm{CNSs}$; we will refer to this set as novel-St neurons. In addition, the mutant CNSs were also dotted with several other fluorescent neurons with low and variable intensities. We could not reliably define their patterns in the third instar CNSs.

Figure $4, A$ and $B$, shows the prominent $C F$ neurons in the mutant and wild-type $\mathrm{Sb}-\mathrm{Th}$ region. As shown in Figure $4 A$, in the mutant CNS the CF neuronal pattern in the $\mathrm{Sb}-\mathrm{Th}$ region is dominated by the novel-ST cluster. The novel-ST cluster is composed of a dozen or more irregularly shaped, intensely fluorescent cells. The somata of these neurons appear to be larger and more intensely fluorescent than any other CF neurons in the mutant CNS.

The increase in the total number of fluorescent neurons and a reduction in the resolution of individual fluorescent somata in the mutant CNSs made it difficult to ascertain whether the entire normal- $C F$ neuronal complement was present in the mutant CNSs. These problems were most acute in the medial SbTh region, where the novel-ST neurons dominated the histofluorescence (Fig. 4A). Consequently, the presence or absence of the normal neurons could not be ascertained in the medial SbTh region. The AbU neurons were rarely observed in the mutant CNSs. Sometimes, especially in the early third instar mutant CNS, a set of diffusely fluorescent, irregularly shaped neurons was observed near the midline in the thoracic and abdominal regions. These neurons appeared to be unpaired, but were distinct from the AbU normal-CF neurons and will be referred to as irregular medial (IM) neurons. Neurons in a location similar to the $\mathrm{AbL}$ row were routinely observed (Fig. $4 \mathrm{~A}$ ). The somata of the AbL neurons were small, as in the case of the wild-type.

The perturbed CF pattern is already present in the newly hatched Df Ddc larval CNS

Since the dominant feature of the perturbation in the CF pattern (the novel-ST cluster) was routinely observed in the CNSs dissected from $D f D d c$ third instar larvae, we believe that the altered CF pattern is a specific result of the absence of endogenous DDC activity. The novel-CF neurons observed in the mutant CNSs raise a number of questions regarding the origin of the novel-CF neurons and the identity of the catecholamine(s) responsible for the histofluorescence. When during development does the mutant pattern become evident? Are the novel-CF neurons born in response to the absence of DDC activity, or is some normally occurring subset of neurons showing a change in its qualitative or quantitative chemical constitution?
In order to determine when during development the differences between the mutant and wild-type CF pattern first appear, CNSs from mutant and wild-type pharate larvae, mid-first instar larvae, and second instar larvae were examined.

Pharate larval CNS. The CNS at this early developmental stage is small and fragile (compare first and third instar CNSs in Fig. $1 A$ ), and, in addition, $D f D d c$ neural tissue tends to be extremely sticky, making it difficult to handle and orient. Because of these technical difficulties, the criteria used to interpret the mutant and the wild-type CF patterns in the individual ganglia were simplified. Presence of the novel-ST cluster was considered indicative of the mutant pattern; presence of at least $4 \mathrm{AbL}$ and $\mathrm{AbU}$ neurons was considered indicative of the respective normal patterns. We felt justified in this simplification because, on occasion, when a sample was intact and properly oriented for observation, like the sample depicted in Figure 5, the full complement of neurons was evident.

In wild-type CNSs dissected from newly hatched larvae, all the normal- $C F$ neuronal subsets could be observed (7/7 samples), although the high level of background fluorescence resulted in a somewhat blurred image. The mutant CNSs were dissected from larvae that were still in the embryonic membranes at a time when the wild-type would normally hatch. The histofluorescence in the mutant samples was low, but when $\mathrm{CF}$ neurons could be observed, they were of the novel-CF type observed in the mutant CNSs (8/12 samples).

First instar and second instar larval CNS. Figure 5 shows the fluorescence micrograph of the mutant CNS dissected from an early second instar larva. In all the mutant CNS whole-mounts examined at this stage, novel-ST neurons, as well as normal$C F$ neurons (AbL and AbU), were observed (6/6 samples). Similar results were obtained from the mid-first instar mutant $\mathrm{CNSs}$ ( $5 / 5$ samples). In most samples a second set of novel- $C F$ neurons, consisting of 4-6 pairs of paramedial neurons (novel-PM neurons) located close to the midline were observed. The novel$P M$ neurons are depicted in Figure $5 A$. Their location was posterior to the novel-ST cluster and their cell bodies smaller than those of the novel-ST cluster. Figure $5 A$ also shows the AbU set of normal- $C F$ neurons. Figure $5 B$ shows the same wholemount preparation, but the plane of focus is on the dorsally located $\mathrm{AbL}$ set of normal-CF neurons.

Studies of the mutant CNS at different developmental stages led to the following conclusions: (1) The perturbations observed in the mutant CNSs are already present at hatching. The concentration of catecholamine(s) in the novel-CF neurons is close to the detection level at hatching and thereafter remain above 

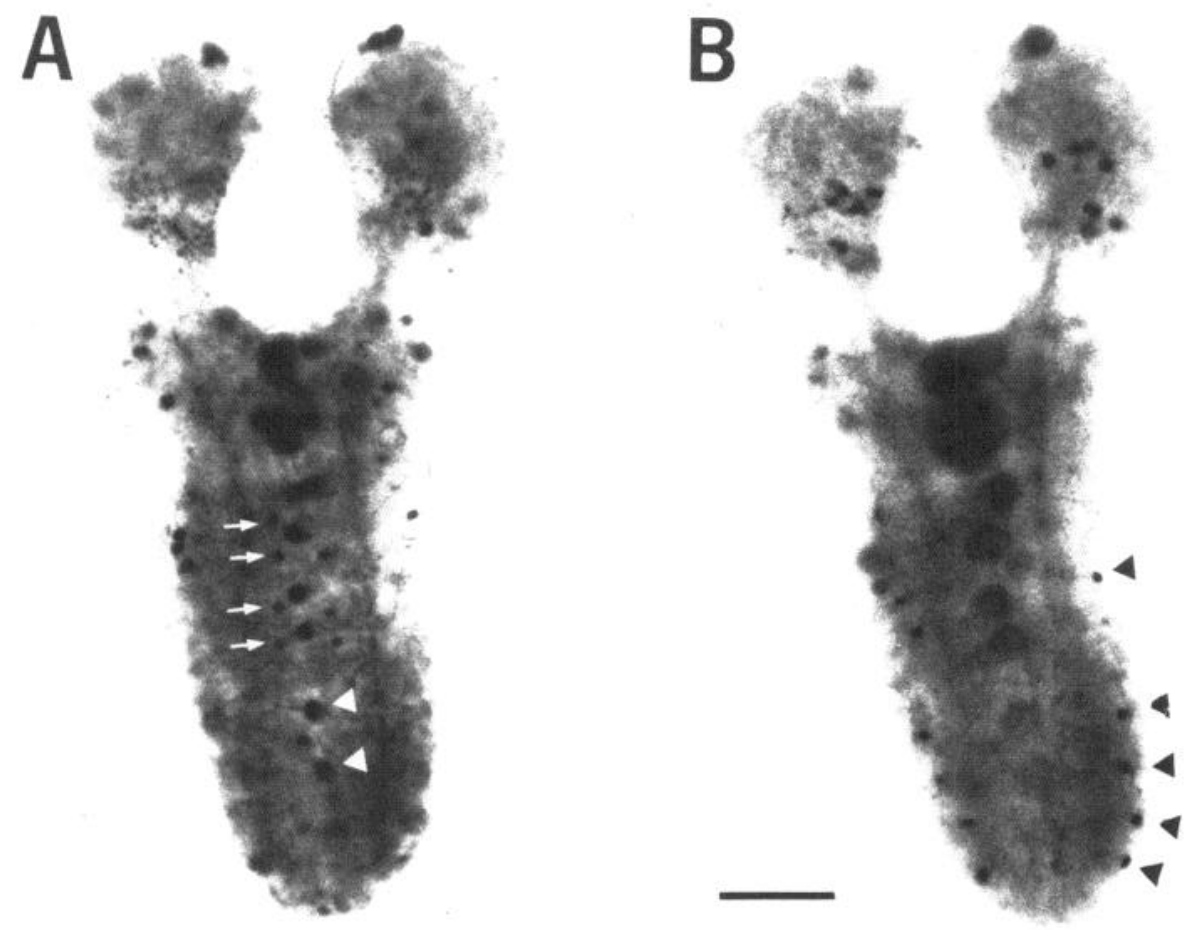

Figure 5. CF neuronal pattern of a $D f D d c$ second instar larval CNS. The prints are a negative image of a fluorescence micrograph. $A$, Photomicrograph showing the novel- $P M$ neurons and the $A b U$ subset of the normal-CF pattern. White arrows point to the left row of novel-PM neurons, which flank the $\mathrm{AbU}$ neurons. Two of the posterior AbU neurons are pointed out by the white arrowheads. $B$, Same whole-mount as in $A$, but the plane of focus is dorsal, showing some of the AbL neurons. The black arrowheads point to the right row of the AbL neurons. Bar, $15 \mu \mathrm{m}$. Axes, same as Figure 1. the detection level throughout the larval developmental period. (2) The catecholamine concentrations within the normal-CF neurons, in the absence of DDC activity, are low at the beginning of the first larval instar. (3) The catecholamine level in the normal-CF neurons in the mutant CNS increases during the first larval instar such that both the novel-CF and the normal$C F$ neuronal patterns are observed during the second half of the first instar and the second larval instar stages. (4) During the third larval instar, the catecholamine level in the normal-CF neurons appears to decrease in the mutant CNS.

\section{Catecholamine-uptake studies}

The embryonic origins of the novel-CF neurons in the mutant CNSs strongly imply that a specific set of neurons is exhibiting a change in its catecholamine composition in the mutant CNS. Presumably, in the wild-type CNS, this set of neurons either does not contain fluorogenic catecholamine(s) or the fluorogenic catecholamine(s) within these neurons do not attain the concentrations required for histofluorescent detection. But in the $D f D d c$ mutant CNSs, they acquire high levels of catecholamine(s).

Although the cellular mechanism(s) by which the fluorogenic amine(s) attains high concentration in the mutant CNSs is not known, the neurons must have a set of properties that distinguish them from other neurons. These neurons may be in some way related to or share common properties with the normal$C F$ neurons. An obvious specific property to test is the selective catecholamine-uptake capability of the monoamine-containing neurons (Axelrod, 1965). The selective ability to take up monoamines has been used to preload cells with fluorogenic amines for better visualization (Flanagan, 1983; Kerkut et al., 1967). In fact, such preloading of neurons with dopamine in the ganglia of the hemipteran insect Rhodnius prolixus results in more reliable detection of the CF neurons, and reveals additional sets of neurons that are normally nonfluorogenic, but can sequester exogenous dopamine (Flanagan, 1983, 1986). Interestingly, nonfluorogenic dopamine-sequestering cells in Rhodnius are also found in the subesophageal and prothoracic region (Flanagan, 1983, 1986).

Catecholamine preloading of third instar larval CNS. CNSs were dissected from third instar wild-type larvae and incubated in exogenous amine at concentrations ranging from $10^{-7}$ to $10^{-3}$ $\mathrm{M}$, washed and then reacted with glyoxylic acid, as described in Materials and Methods. As controls, CNSs were incubated in Ringer's solution alone, washed, and processed in parallel to the experimental samples. Table 2 presents the data on the mean

Table 2. Mean number of neurons observed in normal-CF neuronal subsets ${ }^{a}$

\begin{tabular}{|c|c|c|c|c|c|c|}
\hline \multirow[b]{2}{*}{ Larval stage } & \multirow{2}{*}{$\begin{array}{l}\text { No. of } \\
\text { samples }\end{array}$} & \multirow{2}{*}{$\begin{array}{l}\text { Sb-Th } \\
\mathrm{U}+\mathrm{P}\end{array}$} & \multicolumn{2}{|c|}{$\mathrm{Ab}$} & \multirow{2}{*}{$\begin{array}{l}\text { Th } \\
\text { L }\end{array}$} & \multirow[b]{2}{*}{ Tota } \\
\hline & & & $\mathrm{U}$ & $\mathrm{L}$ & & \\
\hline Third instar ${ }^{b}$ & 13 & 8 & 7 & 13 & 3 & 31 \\
\hline \multicolumn{7}{|l|}{ Third instar } \\
\hline Ringer's (70 min) & 22 & 8 & 7 & 12 & 3 & 31 \\
\hline \multicolumn{7}{|l|}{ Second instar } \\
\hline Ringer's (70 min) & 12 & 6 & 5 & 9 & 3 & 23 \\
\hline
\end{tabular}

${ }^{a}$ The mean was rounded to the closest integer.

${ }^{b}$ Data from Table 1. 

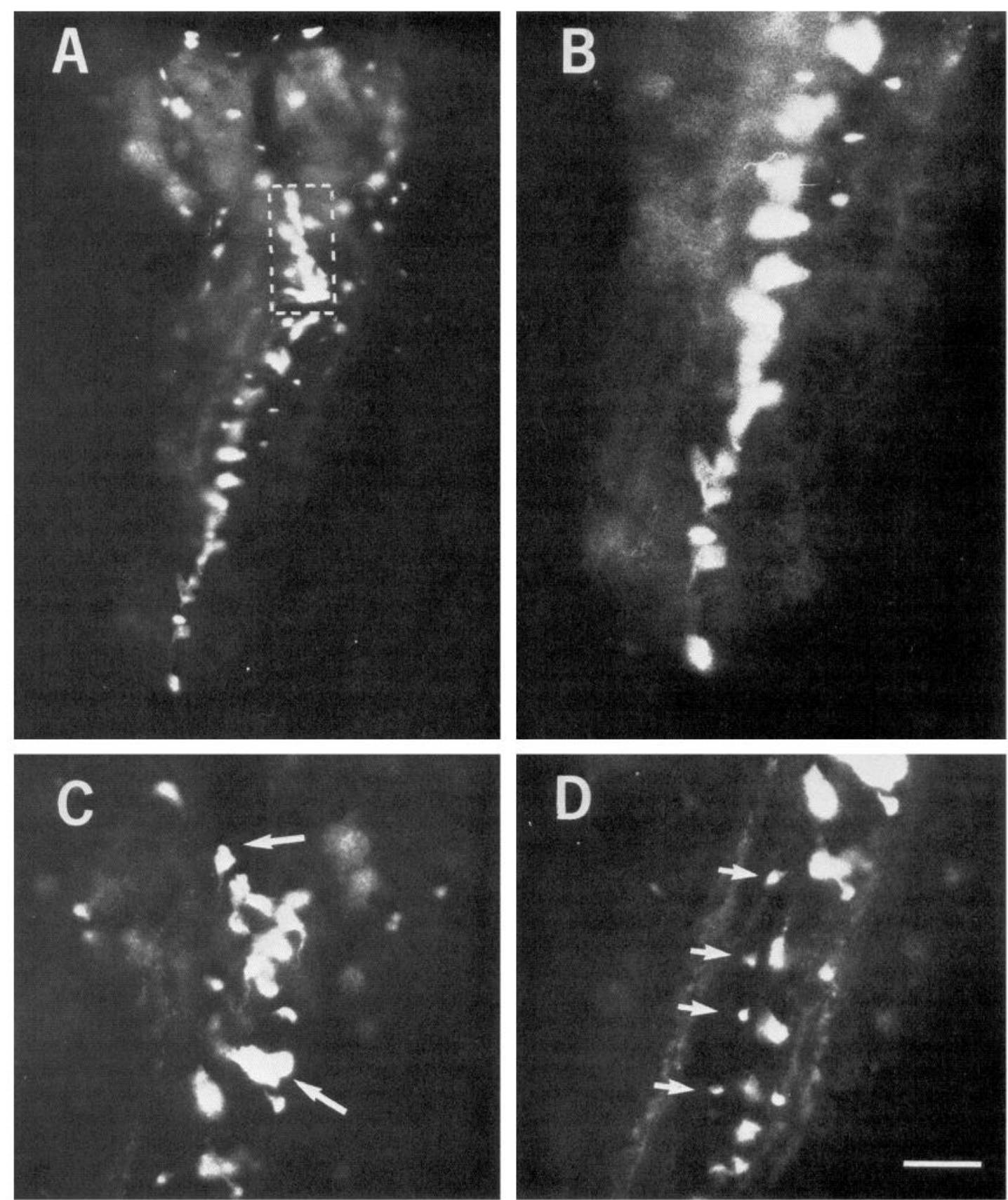

Figure 6. CF neuronal pattern after preloading with L-dopa $\left(10^{-3} \mathrm{M}\right) . A$, Glyoxylic acid-reacted third instar larval CNS whole-mount. Note the additional CF neurons that are normally nonfluorogenic (compare to Fig. $1 A$ ). The white box delineates the $\mathrm{Sb}-\mathrm{Th}$ region. $B$, A higher-magnification photograph of the whole-mount shown in $A$, showing the ventral medial row of CF neurons in the abdominal neuromeres. Note the increased number of somata compared to the AbU subset of the normal-CF neurons shown in Figure $2 A$. Note also the large and irregular shape of the irregular medial (IM) neurons. $C$, The normally nonfluorogenic Sb-Th neurons revealed after preloading with L-dopa. White arrows delineate the $\mathrm{Sb}$-Th region. This photograph is of a different whole-mount sample than that shown in $A$ and $B$. $D$, Photograph of the same whole-mount as in $C$, showing the L-dopa-sequestering paramedial (PM) neurons revealed after preloading with $\mathrm{L}$-dopa. The white arrows point to the PM neurons. Compare the PM neurons in this photograph to the novel-PM neurons shown in Figure $4 A$. Bar, $20 \mu \mathrm{m}$ in $A, 10 \mu \mathrm{m}$ in $B-D$. Axes, as in Figure 1 .

number of CF somata observed in control ganglia. The 22 samples were from 5 different experimental series. The top line shows the mean number of somata in different neuronal groups for the individual ganglia from Table 1. The data from the samples incubated for $70 \mathrm{~min}$ at room temperature (Table 2, line 2) and from those reacted immediately (Table 2, line 1) are very similar, indicating that the normal-CF pattern persists throughout the manipulation.

The CF neuronal patterns in the ventral ganglion of CNSs incubated with tyrosine or norepinephrine at concentrations ranging from $10^{-7}$ to $10^{-3} \mathrm{M}$ were indistinguishable from the control. The CF patterns after incubations with $10^{-7}-10^{-4} \mathrm{M}$ of $\mathrm{L}$-dopa or dopamine were also indistinguishable from the control pattern. But incubations with L-dopa or dopamine at a concentration of $10^{-3} \mathrm{M}$ revealed additional neurons (Fig. $6 \mathrm{~A}$ ). In these CNSs, all the elements of the normal- $C F$ pattern could be identified and, additionally, sets of neurons that are normally nonfluorogenic now fluoresced. Most of the additional fluoro- 
Table 3. $C F$ neuronal sets revealed after preloading wild-type CNS with $L$-dopa or dopamine

\begin{tabular}{llcccc} 
Larval & & \multicolumn{2}{c}{ L-dopa-sequestering neurons } & Normal-CF \\
\cline { 3 - 5 } instar & Amine conc. & Sb-Th & $\begin{array}{l}\text { Para- } \\
\text { medial }\end{array}$ & Irregular & $\begin{array}{l}\text { Norms } \\
\text { neurons }\end{array}$ \\
\hline Third & $10^{-3}$ M L-dopa & $13 / 14$ & $10 / 14$ & $14 / 14$ & $11 / 14$ \\
& $10^{-3}$ M dopamine & $8 / 9$ & $6 / 9$ & $9 / 9$ & $9 / 9$ \\
Second & $10^{-5}$ M L-dopa & $12 / 16$ & $8 / 16$ & $14 / 16$ & $16 / 16$ \\
& $10^{-3}$ M dopamine & $7 / 8$ & $3 / 8$ & $5 / 8$ & $8 / 8$
\end{tabular}

Data presented as nos. of samples in which the CF neuronal set was observed/total no. of samples.

genic neurons were highly reminiscent of the novel-CF neurons observed in the $D f D d c$ CNSs. The normally nonfluorogenic neurons able to sequester L-dopa or dopamine are described below and will be referred to as "L-dopa-sequestering neurons." We have focused on those neuronal sets that were reliably observed and had readily recognizable patterns.

1. Medial subesophageal-thoracic neurons. These neurons were located in the $\mathrm{Sb}$ - Th region near the dorsal midline. Their location, shape, and overall appearance were similar to those of the novel-ST cluster in the mutant CNS. Similar to the mutant CNS, these neurons constitute the most conspicuous feature of the CF pattern after preloading with the amine (Fig. $6, A, C$ ).

2. Paired paramedial neurons. Four to 6 pairs of neurons located close to the midline were observed (Fig. 6D). These neuronal pairs were similar to the novel- $P M$ neuronal pairs observed in mutant CNSs with respect to their location, overall shape, and number (compare Fig. $5 A$ to Fig. $6 D$ ).

3. Latent irregular medial neurons. A set of diffusely fluorescent, irregularly shaped neurons was observed near the midline in the thoracic and abdominal regions (approximately 20-25). These neurons were similar to the previously mentioned novel$I M$ neurons observed in the early third instar mutant CNS (Fig. $6, A, B)$.

The pattern of $\mathrm{CF}$ in individual ventral ganglia that were incubated in exogenous amine was hard to define with the same rigor as the normal-CF pattern in the controls because of the increased number of fluorogenic neurons in the CNS (Fig. 6, $A-$ $D$ ) and a more variable degrec of fluorescence in both the individual neurons and the background. Therefore, simpler qualitative criteria were used in their analysis. The presence of some $\mathrm{AbL}$ and $\mathrm{AbU}$ neurons was considered indicative of normal$C F$ neurons. Each ganglion was also inspected for the presence of neurons corresponding to the 3 neuronal subgroups evidenced after preloading with the amine, as described above. Table 3 provides a summary of CF neurons observed in the wild-type third instar ventral ganglia after incubations in $10^{-3} \mathrm{M} \mathrm{L}$-dopa or dopamine. The major point that emerges from the data presented in Table 3 is that the normal-CF neurons, as well as the neurons specifically observed only after incubation in L-dopa or dopamine, were consistently observed.

The additional $\mathrm{CF}$ neurons observed after preloading with the amine in the third instar CNSs were revealed only when high concentrations of the exogeneous amines $\left(10^{-3} \mathrm{M}\right)$ were used. The high concentrations were probably required because of the inability of the amines to pass freely through the sheath that envelops the CNS. Two observations strengthen this possibility. In broken samples, some additional CF neurons were observed at lower amine concentrations, but only in the vicinity of the break, and we have previously shown that the older third instar CNSs are less permeable than younger CNSs to exogenously added serotonin (Vallés and White, 1986). Therefore, amine-preloading experiments were performed with second larval instar CNS to test whether additional CF neurons could be revealed after incubation at lower concentrations of the amines.
L-Dopa preloading of second instar larval CNS. CNSs were dissected from second instar larvae incubated in $\mathrm{L}$-dopa $\left(10^{-6}\right.$ $10^{-3} \mathrm{M}$ ) or dopamine $\left(10^{-3} \mathrm{M}\right)$ and processed as described above. Control CNSs, incubated in Ringer's alone, were processed in parallel. The results of the control samples are summarized in Table 2, and the results of the experimental sample are summarized in Table 3 . The normal-CF pattern evidenced in the control samples in the second instar larval ganglia was somewhat more variable than the control third instar ganglia (Table 3 ). The mean number of neurons observed in 12 control samples was 24. The deviations from the expected CF pattern were always in absence of neurons.

Incubation in dopamine at a concentration of $10^{-3} \mathrm{M}$ gave a pattern similar to that seen with the third instar CNSs incubated with dopamine or L-dopa. Incubations in L-dopa, however, revealed the additional sets of CF neurons reproducibly, at a lower concentration $\left(10^{-5} \mathrm{M}\right)$. At a higher level of L-dopa $\left(10^{-3} \mathrm{M}\right)$, nonspecific staining of the tissue was routinely observed in these second instar samples (8/10 samples). The nonspecific staining was characterized by the presence of some degree of fluorescence, coming from all cells in the tissue. Even in these samples showing nonspecific fluorescence in all neurons, the neuronal cluster in the $\mathrm{Sb}$ - $\mathrm{Th}$ region that was revealed after preloading with the amine was intensely fluorescent, while the remaining CF neuronal sets were frequently obscured. These data suggest that the second instar sheath may be more permeable to L-dopa than dopamine, or that L-dopa uptake might be more efficient at this stage. Another possibility is that dopamine is more readily converted to a nonfluorogenic product than L-dopa.

In summary, the studies of wild-type CNSs incubated in exogenous L-dopa or dopamine at different amine concentrations lead to the following conclusions: (1) In the control samples incubated in Ringer's alone, the normal- $C F$ pattern persists (Table 2). (2) Normal-CF neurons are still fluorogenic after incubations with L-dopa or dopamine. (3) Incubations with Ldopa or dopamine reveal a population of neurons that is normally nonfluorogenic but is capable of sequestering L-dopa and dopamine. (4) The subpatterns observed within the population of the additional CF neurons revealed after preloading are similar, in localization and overall appearance, to the novel-CF neurons observed in the mutant $D f D d c$ CNSs, although all the subpatterns may not be discerned in an individual mutant CNS.

\section{Discussion}

\section{CF neurons}

In this study, the in situ detection of catecholamines is based entirely on glyoxylic acid-induced histofluorescence and, as such, all its results are subject to the limitations imposed by this methodology. As was discussed at the beginning of Results, the fluorogenic catecholamine(s) in any given neuron cannot be specifically identified. It is reasonable to assume that at least some and probably most of the normal-CF neuronal subsets in Dro- 
sophila contain dopamine (Livingstone and Tempel, 1983). But it is possible that certain neurons may instead be norepinephrine-containing, or may contain another, as-yet-unidentified, fluorogenic catecholamine. A second point is that only neurons with fluorogenic catecholamine concentrations above a certain threshold are revealed by this assay.

Similar to what has been observed in other invertebrates, the normal-CF neuronal pattern is stereotypic in Drosophila larval CNS (Flanagan, 1983; Lent, 1982). CF neurons were observed in the brain lobes, the subesophageal ganglion, the 3 thoracic neuromeres, and in most abdominal neuromeres. Comparison of the catecholamine-containing neurons to the previously characterized serotonin-containing neurons in the Drosophila ventral ganglia shows that the 2 sets are nonoverlapping (Vallés and White, 1986). The current evidence suggests that octopamine is present in Drosophila CNS (Livingstone and Tempel, 1983), but as yet the octopamine-containing neurons have not been directly identified.

Comparison of the CF neuronal pattern in Drosophila ventral ganglion to the pattern described for the ventral cord of the hemipteran Rhodnius prolixus (Flanagan, 1983, 1986) reveals remarkable similarities. For example, in Rhodnius the subesophageal ganglion and the prothoracic ganglion each contains a cluster of 3 intensely fluorescent cells. The cluster is composed of an unpaired medial cell and a pair close to the midlinesimilar to what is observed in the homologous ganglia in Drosophila. One distinction between the patterns in Drosophila and Rhodnius is that these cells are located in the ventral cellular rind in the fly CNS, whereas the Rhodnius cells are reported as being dorsally located (Flanagan, 1983, 1986).

\section{Catecholamine-containing neurons differentiate in the absence} of $D D C$ activity

In Drosophila, the gene $D d c$ encodes an aromatic amino acid decarboxylase present in the brain and required in the synthesis of serotonin and dopamine (Livingstone and Tempel, 1983). Furthermore, it appears to be the only decarboxylase used in the synthesis of serotonin, judging by the complete absence of serotonin-immunoreactivity in the $D f D d c$ mutant CNSs (Vallés and White, 1986). The methodology used in this study did not permit direct assertion of dopamine absence in the CF neurons in the mutant CNSs.

In the wild-type CNS, the normal-CF neurons are routinely observed in the newly hatched larval CNS. In the mutant CNSs, the normal- $C F$ neurons are not observed at the newly hatched stage but are evident several hours later, by the mid-first instar stage. We suggest that the fluorogenic amine in the normal-CF neurons that is found in the mutant CNSs is likely to be L-dopa, which will accumulate when conversion to dopamine is blocked (Fig. 3). The normal-CF neurons in the mutant CNSs seem to persist throughout the larval stages. These observations imply that the catccholaminc-containing ncurons differentiatc in the absence of DDC activity. The situation with the normal catecholamine-containing neurons appears to be analogous to that of the serotonin-containing neurons, which also persist and express at least some cell-type-specific properties (Vallés and White, 1986).

\section{Novel-CF neurons are observed in the Df Ddc CNSs}

A novel set of fluorogenic neurons is observed in the mutant CNSs. Interestingly, in the mutant $\mathrm{CNS}$, the novel- $C F$ neurons fluoresce before the normal-CF neurons. Since the novel- $C F$ neurons are present in the newly hatched larvae, they have "cell birthdays" during the embryonic period. It is highly unlikely that the novel neurons are born in response to the DDC deficiency, since apparently the same neurons can be labeled in wild-type CNSs (see below) and the first expression of the zygotic $D d c$ gene activity occurs at around $16 \mathrm{hr}$ of embryogenesis (Beall and Hirsh, 1984; Kraminsky et al., 1980), which is well after the period of active cell division in the embryonic CNS (Poulson, 1950). Although the cell-division pattern in the mutant embryo has not been studied, it seems reasonable to suggcst that a set of neurons that is normally nonfluorogenic in the wildtype becomes fluorogenic in the mutant CNS.

\section{Normally nonfluorogenic neurons can take up L-dopa or dopamine}

The studies of the wild-type CNSs that are preloaded with L-dopa or dopamine demonstrate that a set of normally nonfluorogenic neurons can take up exogenous L-dopa and dopamine. Among the CF neurons observed after incubations in exogenous L-dopa or dopamine are neuronal subsets that resemble the normal-CF neurons as well as the novel-CF neurons.

We compared the $C F$ neuronal patterns revealed after preloading Drosophila and Rhodnius with the amine in their subesophageal and prothoracic ganglia (Flanagan, 1986). Analogous to the case of the normal-CF neurons, where remarkable similarities are observed, the patterns after dopamine uptake also showed striking similarities. In Rhodnius CNS, as in Drosophila CNS, after incubation in exogenous dopamine normally nonfluorogenic neurons became fluorogenic. A major cluster of these normally nonfluorogenic neurons that became fluorogenic in the Rhodnius CNS was located near the dorsal midline in the subesophageal ganglion and in the prothoracic ganglion. Flanagan (1983) has suggested that these midline subesophageal-prothoracic neurons may be octopaminergic because of their similarity to the well-characterized midline octopaminergic neurons in the locust and because they also can be stained with the vital dye neutral red.

\section{Accumulation of catecholamine in the novel-CF neurons in the mutant CNSs}

The circumstantial evidence presented here suggests that subsets of the normally nonfluorogenic neurons capable of sequestering L-dopa or dopamine in the mulant $D f D d c$ CNSs appear as the novel- $C F$ neurons. At present, we can only speculate about the mode of accumulation of the fluorogenic amine in the novel$C F$ neurons.

It is possible that the nonfluorogenic neurons that are able to sequester L-dopa or dopamine normally synthesize a catecholamine that is nonfluorogenic or that the levels of fluorogenic catecholamine are normally below the level of detection. In the mutant, the block in the pathway leads to accumulation of a fluorogenic amine, L-dopa. The implication would be that these neurons in the wild-type contain tyrosine hydroxylase and DDC (see Fig. 3). Immunological tools could potentially be used to test for the presence of these 2 synthetic enzymes.

An alternate possibility is that the tyrosine hydroxylase gene might be activated in the novel-CF neurons in the mutant, leading to conversion of tyrosinc to $L$-dopa. A third scenario depends on the assumption that the normally nonfluorogenic neurons that are able to sequester L-dopa or dopamine are octopaminecontaining. The $D d c$ gene does not seem to be responsible for the conversion of tyrosine to tyramine in Drosophila (Livingstone and Tempel, 1983). It is possible that in the mutant CNSs, catecholamines may be synthesized, using tyramine as a precursor in octopamine-containing neurons, by an alternate biochemical pathway.

A fourth plausible explanation is that the high levels of $L$-dopa are acquired in a nonautonomous fashion by the novel-CF neurons serving as a sink for the excess L-dopa secreted by other neurons or tissues in the mutant. In support of this hypothesis is the observation that in the wild-type CNS there appear to be neurons that can take up L-dopa in homologous positions to the novel-CF neurons in the mutant. However, since the novel-CF neurons already fluoresce in the newly hatched larval CNS, this 
hypothesis would imply that high levels of L-dopa are accumulated within a few hours in the absence of DDC activity and that L-dopa is available to the neurons.

To conclude, we have demonstrated that the genetic deficiency of DDC enzyme activity leads to perturbation of the normal-CF neuronal pattern, and we have characterized the conspicuous aspects of these perturbations. We have also provided evidence that normally nonfluorogenic neurons in the wild-type CNS in positions homologous to the novel-CF neurons in the mutant CNSs can sequester L-dopa or dopamine.

\section{References}

Axelrod, J. (1965) The metabolism, storage and release of catecholamine. Recent Prog. Horm. Res. 21: 597-622.

Beall, C. J., and J. Hirsh (1984) High levels of intron-containing RNAs are associated with expression of the Drosophila dopa decarboxylase gene. Mol. Cell. Biol. 4: 1669-1674.

Budnik, V., A. M. Vallés, and K. White (1985) Histofluorescence of catecholamine-containing neurons in Drosophila. Soc. Neurosci. Abstr. 11: 944.

Flanagan, T. R. J. (1983) Monoaminergic innervation in a hemipteran nervous system: A whole-mount histofluorescence survey. In Functional Neuroanatomy, N. J. Strausfeld, ed., pp. 317-329, Springer, New York.

Flanagan, T. R. J. (1986) Serotonin-containing catecholamine-containing and dopamine-sequestering neurons in the ventral nerve cord of the hemipteran Rhodnius prolixus. J. Insect Physiol. 32: 17-26.

Gilbert, D., J. Hirsh, and T. R. F. Wright (1984) Molecular mapping of a gene cluster flanking the Drosophila dopa decarboxylase gene. Genetics 106: 679-694.

Greenspan, R. J. (1980) Mutations of choline acetyltransferase and associated neural defects in Drosophila melanogaster. J. Comp. Physiol. 137: 83-92.

Greenspan, R. J., J. A. Finn, and J. C. Hall (1980) Acetylcholinesterase mutants in Drosophila and their effects on the structure and function of the central nervous system. J. Comp. Neurol. 189: 741-774.

Haydon, P. G., D. P. McCobb, and S. B. Kater (1984) Serotonin selectively inhibits growth cone motility and synaptogenesis of specific identified neurons. Science 226: 561-564.

Hökfelt, T., O. Johansson, and M. Goldstein (1984) Chemical anatomy of the brain. Science 225: 1326-1334.

Kerkut, G. A., C. B. Sedden, and R. J. Walker (1967) Uptake of dopa and 5-hydroxytryptophan by monamine-forming neurons in the brain of Helix aspersa. Comp. Biochem. Physiol. 23: 159-162.
Klemm, N. (1980) Histochemical demonstration of biogenic monoamincs (Falck-Hillarp method) in the insect nervous system. In Neuroanatomical Techniques-Insect Nervous System, N. J. Strausfeld and T. A. Miller, eds., pp. 52-69, Springer, New York.

Kraminsky, G. P., W. C. Clark, M. A. Estelle, R. D. Gietz, B. A. Sage, J. D. O'Connor, and R. B. Hodgetts (1980) Induction of translatable mRNA for dopa decarboxylase in Drosophila: An early response to ecdysterone. Proc. Natl. Acad. Sci. USA 77: 4175-4179.

Lent, C. M. (1982) Fluorescent properties of monoamine neurons following glyoxylic acid treatment of intact leech ganglia. Histochemistry $75: 77-89$.

Lindvall, O., and A. Björklund (1974) The glyoxylic acid fluorescence histochemical method: A detailed account of the methodology for visualizing of catecholamine neurons. Histochemistry 39: 97-127.

Lindvall, O., A. Björklund, and L. A. Svensson (1974) Fluorophore formation from catecholamines and related compounds in the glyoxylic acid fluorescence histochemical method. Histochemistry 39: 197227.

Lindvall, O., A. Björklund, B. Falck, and L. A. Svensson (1975) Combined formaldehyde and glyoxylic acid reactions. I. New possibilities for microspectrofluorometric differentiation between phenylethylamines, indolylethylamines and their precursor amino acids. Histochemistry $46: 27-52$.

Livingstone, M. S., and B. L. Tempel (1983) Genetic dissection of monoamine neurotransmitter synthesis in Drosophila. Nature 303: $67-70$.

McCobb, D. P., P. G. Haydon, and S. B. Kater (1985) Dopamine: An additional regulator of neurite outgrowth in Helisoma. Soc. Neurosci. Abstr. 11: 761.

Mercier, A. J., P. G. Haydon, and S. B. Kater (1985) A rolc for serotonergic neurons in the regulation of specific electrical synapses in Helisoma. Soc. Neurosci. Abstr. 11:614.

Poulson, D. F. (1950) Histogenesis, organogenesis and differentiation in the embryo of Drosophila melanogaster. In Biology of Drosophila, M. Demerec, ed., pp. 168-174, Wiley, New York.

Vallés, A. M., and K. White (1986) Development of serotonin-containing neurons in Drosophila mutants unable to synthesize serotonin. J. Neurosci. 6: 1482-1491.

Wright, T. R. F., R. B. Hodgetts, and A. F. Sherald (1976) The genetics of dopa decarboxylase in Drosophila melanogaster. I. Isolation and characterization of deficiencies that delete the dopa-decarboxylasedosage-sensitive region and the alpha-methyl-dopa-hypersensitive locus. Genetics 84: 267-285. 